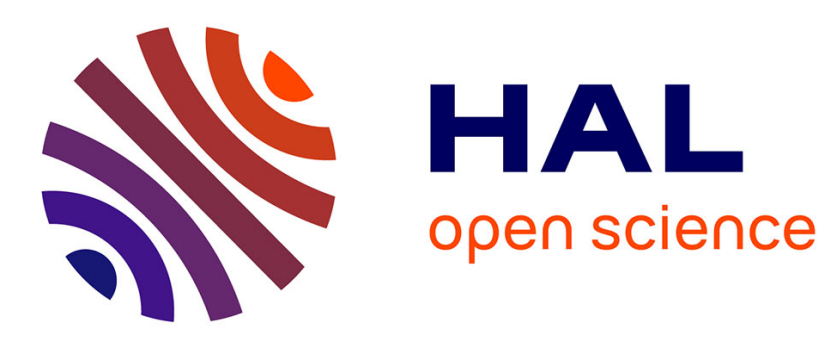

\title{
A Critical Overview of the Most Recent Logics of Grounding
}

Francesca Poggiolesi

\section{To cite this version:}

Francesca Poggiolesi. A Critical Overview of the Most Recent Logics of Grounding. Francesca Boccuni, Andrea Sereni Objectivity, Realism and Proof, 318, Springer, pp.291-309, 2016, Boston Studies in the Philosophy and History of Science, 978-3-319-31642-0. 10.1007/978-3-319-31644-4_15 . hal01344256

\section{HAL Id: hal-01344256 https://hal.science/hal-01344256}

Submitted on 11 Jul 2016

HAL is a multi-disciplinary open access archive for the deposit and dissemination of scientific research documents, whether they are published or not. The documents may come from teaching and research institutions in France or abroad, or from public or private research centers.
L'archive ouverte pluridisciplinaire HAL, est destinée au dépôt et à la diffusion de documents scientifiques de niveau recherche, publiés ou non, émanant des établissements d'enseignement et de recherche français ou étrangers, des laboratoires publics ou privés. 


\title{
A Critical Overview of the Most Recent Logics of Grounding
}

Francesca Poggiolesi

Université Paris 1 Panthéon-Sorbonne,

CNRS, ENS,

UMR 8590 IHPST -

Institut d'Histoire et de Philosophie

des Sciences et des Techniques

poggiolesi@gmail.com, +33629721836

\begin{abstract}
In this paper our aim is twofold: on the one hand, to present in a clear and faithful way two recent contributions to the logic of grounding, namely Correia (2014), and Fine (2012a); on the other hand, to argue that some of the formal principles describing the notion of grounding proposed by these logics need to be changed and improved.
\end{abstract}

Keyword grounding, logic, proof theory

\section{Introduction}

The last decade has witnessed an increasing interest for the concept of grounding. Grounding is either described as a "special sort of non-causal priority" Correia and Schnieder (2012) or as an "objective relation which is explanatory in nature" Correia (2014). Grounding is typically conveyed by the linguistic expression 'because', but other expressions like 'in virtue of' and 'due to' can also serve the purpose. Some examples of grounding sentences are the following:

- Albert did not go to school today because he was sick;

- John is tall and thin because John is tall and John is thin;

- these two apples resemble to each other because they have the same shape and the same color.

As is clear even at the first glance, these three sentences share a common structure: each of them contains the expression because and each of them can be divided into an antecedent, i.e. what comes after the because ("Albert was sick", "John is tall and John is thin" and "the apples have the same shape and the same color"), and a consequent, i.e. what comes before the because ("Albert did not go to school", "John is tall and thin", "these two apples resemble to each other", respectively). In each case we can we say that the consequent is determined, or explained or accounted by the antecedent. In other worlds, in each of the sentences listed above, the antecedent constitutes the reason why, or the ground of the consequent.

Most of the work on grounding is metaphysical (e.g. see Betti (2010); Daily (2012); Schaffer (2009)). Beside this kind of study, two other types of research, that are equally worthy of attention, has been carried out: on the one hand, there exists a rising number of papers dedicated to the history of the notion of grounding, in particular to Bernard Bolzano, a precursor in the study of 


\section{Some standard distinctions concerning the no- tion of grounding}

We will use this section to introduce three familiar distinctions concerning the concept of grounding: the first is that between full and partial grounding, the second is the distinction between immediate and mediate grounding; the third is the distinction between strict and weak grounding. These distinctions have been defined and used by different authors (e.g. see Bolzano (1973); Correia and Schnieder (2012)); here we introduce them in the form proposed by Fine Fine (2012a), which seems to us the form most commonly accepted in the contemporary literature.

Let us start with the distinction between full and partial grounding. A set of truths $M$ is a full ground of the truth $C$ if $M$ is sufficient to explain the truth $C$; A is a partial ground of $C$ if $A$ on its own, or with some other truths, is a full ground of $C$.

Thus, given that $\mathrm{A}, \mathrm{B}$ is a full ground for $A \wedge B$, each of $\mathrm{A}$ and $\mathrm{B}$ will be a partial ground for $A \wedge B$. Each will be relevant to the grounding of $A \wedge B$, even though neither may be sufficient on its own. (Fine, 2012a, p. 50)

Let us now pass to the second distinction, the one between immediate and mediate grounding. $\mathrm{A}$ is an immediate ground of $\mathrm{C}$ if $\mathrm{C}$ may be obtained from $\mathrm{A}$ by means of a single grounding step; $\mathrm{A}$ is a mediate ground of $\mathrm{C}$ if $\mathrm{C}$ is obtained from A by appropriately chaining immediate grounding steps. Thus, while $A \wedge B$ is immediately grounded in $A$ and $B,(A \wedge B) \wedge C$ is only mediately grounded in $A$ and $B$ and $C$.

Before passing to the third and last distinction concerning the grounding concept, let us underline the following point, which will be important later on. We have identified four different types of ground: full and immediate, full and mediate, partial and immediate and partial and mediate. According to the way they have been defined, these types of ground are not pairwise disjoint, i.e. given a set of truths $M$ and a truth $C$ there might be more than one type of grounding relation that intervenes between $M$ and $C$. This is clearly demonstrated by two simple examples. Consider first of all the two truths $A$ and $A \vee B$; according to the definitions above, $A$ is both the full and immediate, but also the partial and immediate ground of $A \vee B$. A is the immediate ground of $A \vee B$ because $A \vee B$ can be obtained from $A$ thanks to an unique grounding step. $A$ is both a full and a partial ground of $A \vee B$ because $A$ is sufficient, but also relevant, to guarantee the truth of $A \vee B$.

Now consider the two truths $A$ and $A \vee(A \vee A)$. According to the definitions above, $A$ and $A \vee(A \vee A)$ enjoy all four types of grounding relation. $A$ is at the same time the full and the partial ground of $A \vee(A \vee A)$ for reasons analogous to the ones mentioned for $\mathrm{A}$ and $A \vee B$. As for $A$ being both immediate and mediate ground of $A \vee(A \vee A)$, we can consider the following explanation 
The truth that $\mathrm{A}$, for example, is a ground for $A \vee(A \vee A)$. It is furthermore an immediate ground for $A \vee(A \vee A)$ since $A$ in its capacity as a left disjunct, so to speak, is not a mediated ground for $A \vee(A \vee A)$. However, $\mathrm{A}$ is an immediate ground for $A \vee A$ and $A \vee A$ is an immediate ground for $A \vee(A \vee A)$; and so A also stand in a mediated relationship of ground to $A \vee(A \vee A)$. (Fine, 2012a, p. 51)

Let us now pass to the third and last distinction concerning grounding that is of interest in this paper. A relation of grounding is strict when it does not allow a truth to partially ground itself; a relation of grounding is weak when it does. Traditionally (e.g. Bolzano (1996)) only strict grounding is taken into account; the relation of weak grounding has been introduced in the recent literature.

In Sections 4 and 5 we will present the logics introduced in Fine (2012a) and Correia (2014), respectively. Instead of considering these logics in the framework of the first-order language, which is the one used by their authors, we will only focus on the framework of the propositional language; moreover, we will restrict the logics of Fine and Correia to the only notion of (weak or strict) full and immediate grounding. There are several reasons for adopting these restrictions. First of all, we do not want the paper to be burdened with too many technical notions and thus we believe that some simplifications are appropriate. Secondly, both the domain of propositional language and the concept of (weak or strict) full and immediate grounding are already rich and fertile enough to inspire reflexions. Thirdly, we privilege the concept of (weak or strict) full and immediate grounding over the others since, as underlined by Bolzano (1973); Tatzel (2002), it is central and, by dealing with it, one really touches the hearth of the matter.

Note that no choice is made with regard to the distinction between strict and weak grounding. Despite the fact that some scholars, like de Rosset (2013), have criticized the notion of weak grounding and we are quite sympathetic to this position, we need both the notions of strict and weak grounding in order to introduce some salient features of the logics of Fine and Correia (e.g. without the weak notion of grounding the elimination rules of Fine's logic could not be introduced.) This is the reason why in this case we have not adopted any restriction.

\section{Grounding as a proof-theoretic notion}

As already emphasized in the introduction, grounding is a deep and complex concept that is and has been studied from several different perspectives, e.g. metaphysical, historical, logical. In this paper we will take a point of view which has recently received a renovated attention (e.g. see Rumberg (2013); Tatzel (2001)) and that could be classified as proof-theoretical: the logics of Fine and Correia will thus be evaluated against such a background. We will use the rest of the section to illustrate the proof-theoretic conception of grounding 
and justify its use in the critical analysis of the logics that can be found in Correia (2014) and Fine (2012a).

In 1935 the logician Gherard Gentzen introduced the calculus of natural deduction. The calculus of natural deduction is a formal system, just like a Hilbert system, where the focus does not go on axioms but on rules. The rules of natural deduction are typically many and can be divided into introduction and elimination rules: each of the former introduces a different logical connective, while each of the latter eliminates a different logical connective. By means of these rules, one can construct derivations that have the form of trees whose leaves correspond to assumptions and whose root corresponds to the conclusion (for a detailed introduction to the calculus of natural deduction see Troelstra and Schwichtenberg (1996)).

Many interesting theorems concerning the calculus of natural deduction can be proved. First of all it can (and should) be shown that a calculus of natural deduction is sound and complete with respect to a certain semantics or a corresponding Hilbert system. Secondly, given a calculus of natural deduction, it can be proved that several rules are admissible and others are derivable in it. Let us remind the reader (since it will prove useful in what follows) that a rule $\mathcal{R}$, that does not belong to a calculus of natural deduction $\mathbf{N}$, is said to be admissible in $\mathbf{N}$ if, whenever there exists a derivation in $\mathbf{N}$ of the premise(s) of $\mathcal{R}$, then there also exists a derivation in $\mathbf{N}$ of the conclusion of $\mathcal{R}$, that does not contain any application of $\mathcal{R}$. A rule $\mathcal{R}$, that does not belong to a calculus of natural deduction $\mathbf{N}$, is said to be derivable in $\mathbf{N}$ if a derivation from the premisses of $\mathcal{R}$ to the conclusion of $\mathcal{R}$ can be constructed in $\mathbf{N}$.

Thirdly and finally, given a calculus of natural deduction, the famous normalization theorem can (and should) be established. This theorem can be roughly explained in the following way. Consider a derivation that contains at least one formula that is neither one of its premisses, nor its conclusion, nor a subformula of its conclusion; such a derivation contains a redundancy, or, in other words, it is a non-analytic derivation. The normalization theorem ensures that any derivation that can be constructed in a calculus of natural deduction is either analytic or can be effectively transformed into an analytic one. The importance of the normalization theorem and of analytic derivations has a long and venerable history that starts with Aristotle (e.g. see Paoli (1991)), pass through Gentzen (1935) and continues today (e.g. see Avron (1991); Indrezejczak (1997); Poggiolesi (2012); Prawitz (1965)): the normalization theorem is by now a cornerstone result of proof theory and analytic derivations represent a paradigm that can hardly be ignored.

Let us now move back to the concept of grounding and consider the link between this concept and the calculus of natural deduction that has so far captured our attention. For many illustrious scholars (e.g. Betti (2010); Bolzano (1996); Casari (1987); Sebestik (1992)), the relationship is simple: grounding is nothing but a special sort of derivability; more precisely, grounding proofs should be seen as particular types of derivations, which reveal ontological hierarchies where truths have been arranged. In this sense grounding is a proof-theoretic notion. If we embrace such a view, as we do in the present paper, there are 
consequences that cannot be neglected. First of all, the formalization of the grounding notion will need to be accomplished by means of a calculus comparable to the one of natural deduction; moreover, such a calculus for grounding will need to satisfy and respect many (if not all) of the properties that have been put forward for the standard calculus of natural deduction. Amongst these, the analyticity property, whose centrality has been emphasized above, will need to keep on playing a special role: grounding chains, as peculiar derivations, will need to be analytic. It is interesting to notice that Bernard Bolzano, the founding father of the theory of grounding, stressed the importance of the analyticity property for grounding chains for reasons independent from the recent developments in contemporary proof theory (see Paoli (1991)).

Correia (2014) and Fine (2012a) present a calculus, as well as a semantics, for the notion of grounding. While Correia proves that his calculus is sound and complete with respect to the semantics that he proposes, the semantics of Fine (2012a) is not adequate for his system (Correia and Schnieder, 2012, p.19). In the next sections, we will only concentrate on the calculi introduced by Fine and Correia. As it seems natural to do, we will evaluate these calculi from a proof-theoretic perspective, that is from the perspective that we have presented in this section.

\section{Fine's Logic}

We will use this section to introduce Fine (2012a) logics for the concepts of strict and weak, full and immediate grounding.

Definition 4.1. Let $\mathcal{L}$ be the language composed by:

- propositional atoms: p, q, r, ...

- classical connectives: $\neg, \wedge, \vee$

$->$ (strict, full and immediate grounding), $\geqslant$ (weak, full and immediate grounding)

As metalinguistic symbols we will use the comma, the semi-colon and the parentheses.

Definition 4.2. The propositional formulas of the language $\mathcal{L}$ are defined by means of the following rule:

$$
p|\neg A| A \wedge B \mid A \vee B
$$

The grounding formulas of the language $\mathcal{L}$ are of one of these two types:

$$
\begin{aligned}
& M>A \\
& M \geqslant A
\end{aligned}
$$

where $M$ is a set of propositional formulas and $A$ is a propositional formula. 
Figure 1: Axioms of the Calculus FG.

\begin{tabular}{|c|c|c|c|}
\hline \multicolumn{4}{|l|}{$A>\neg \neg A$} \\
\hline$A, B>A \wedge B$ & $A>A \vee B$ & $B>A \vee B$ & $A, B>A \vee B$ \\
\hline$\neg A>\neg(A \wedge B)$ & $\neg B>\neg(A \wedge B)$ & $\neg A, \neg B>\neg(A \wedge B)$ & $\neg A, \neg B>\neg(A \vee B)$ \\
\hline
\end{tabular}

The calculus FG (for Fine on grounding) is composed by axioms, see Figure 1, logical rules, see Figure 2, and structural rules, see Figure 3; we will analyse each of these components, starting from structural rules. These are three: the rule SW and the rules ID and NID. The rule SW tells us that from the fact that a relation of strict grounding holds between a set of truths $M$ and a truth $A$ we can infer that a relation of weak grounding holds amongst these truths. The rules ID and NID exemplify the difference between strict and weak grounding: the former is irreflexive, the latter is reflexive. Note that in the original calculus created by Fine the structural rules are much more numerous than the ones that appear in Figure 3: they indeed serve to describe the relationships amongst the several types of grounding (partial and immediate, partial and mediate ...) that have been mentioned in Section 2 but are not discussed here.

Let us now move to the logical rules of Figure 2. These rules are called by Fine elimination rules, just as the elimination rules of the classical natural deduction calculus. Note however an important (from a proof-theoretical point of view, see Indrezejczak (1997); Poggiolesi (2010)) difference between the classical rules and the ones introduced by Fine: while each of the former simply eliminates one classical connective a time, each of latter eliminates one or two classical connectives a time, plus it makes us move from strict to weak grounding. Let us emphasize that in the rules $\vee E$ and $(\neg \wedge) E$ the (meta-linguistic symbol) semi-colon is used: it indeed serves to indicate the disjunctive character of the conclusion.

Let us finally comment on the syntactic objects of Figure 1. Whilst Fine call these objects introduction rules, we prefer to call them axioms since none of them involve an inferential step. These axioms describe the strict, full and immediate grounds of the classical connectives; let us note that negation, differently from conjunction and disjunction, is treated only in its interaction with other connectives.

We would like to end this section with two further remarks. The first concerns the notion of derivation in the calculus FG. It is not clear how to adapt the standard definition of derivation to the calculus FG because of the presence of the (meta-linguistic symbol) semi-colon, for which no indication about use is given. ${ }^{1}$

\footnotetext{
${ }^{1}$ The use of the semi-colon, whose interpretation is in disjunctive terms, could lead one to
} 
Figure 2: Logical Rules of the Calculus FG.

\begin{tabular}{|c|c|}
\hline$\frac{M>\neg \neg A}{M \geqslant A} \neg E$ & \\
\hline$\frac{M>A \wedge B}{M \geqslant\{A, B\}} \wedge E$ & $\frac{M>A \vee B}{M \geqslant\{A, B\} ; M \geqslant B ; M \geqslant A} \vee E$ \\
\hline$\frac{M>\neg(A \wedge B)}{M \geqslant\{\neg A, \neg B\} ; M \geqslant \neg B ; M \geqslant \neg A}(\neg \wedge) E$ & $\frac{M>\neg(A \wedge B)}{M \geqslant\{\neg A, \neg B\}}(\neg \vee) E$ \\
\hline
\end{tabular}

Figure 3: Structural Rules of the Calculus FG.

$$
\begin{aligned}
& \frac{M>C}{M \geqslant C} \text { SW } \\
& \overline{A \geqslant A} \text { ID } \quad \frac{M, A>A}{\perp} \text { NID }
\end{aligned}
$$

The second remark concerns the rule of amalgamtion, which has the following form:

$$
\frac{M_{1}>C, M_{2}>C, \ldots}{M_{1}, M_{2}, \cdots>C} \text { am }
$$

The rule basically says that the strict grounds of a given truth can be amalgamated or combined into a single ground.

It is not usual to include this rule among the rules for ground. But the plausibility of the rules from which it can be derived provides a strong argument for its adoption; and I doubt there is a simple and natural account of the logic of ground that can do without it. (Fine, 2012a, p. 57)

It follows from amalgamation that there always is a maximum full and immediate ground for a grounded truth $C$ : if $M>A$, then there is a $\mathrm{N}$ such that

think of hypersequents (e.g. see Poggiolesi (2008, 2013)). Despite the analogous interpretation, the calculus of Fine and hypersequent calculi are different. In hypersequent calculi not only do we have external structural rules that tell us how to deal with the semi-colon, but also the logical rules are general enough to cover the whole hypersequent object. None of these features is present in FG and this is the reason why it is to hard to figure out how to adapt the standard notion of derivation to this calculus. 
Figure 4: Logical Rules of the Calculus CG.

\begin{tabular}{ccc}
\hline$\frac{A}{\neg \neg A} \neg \neg I$ \\
$\frac{A, B}{A \wedge B} \wedge I$ & $\frac{A}{A \vee B} \vee I$ & $\frac{B}{A \vee B} \vee I$ \\
$\frac{\neg A}{\neg(A \wedge B)}(\neg \wedge) I$ & $\frac{\neg B}{\neg(A \wedge B)}(\neg \wedge) I$ & $\frac{\neg A, \neg B}{\neg(A \vee B)}(\neg \vee) I$
\end{tabular}

(i) $N>A$, and (ii) $P \subseteq N$, for all $P$ such that $P>A$. Note that the same holds for the notion of full and mediate ground.

It is easy to see that the rule of amalgamation is admissible in the calculus FG. Indeed its premisses can only be the axioms of FG and the only cases where these axioms have the same conclusion are the disjunctive and the negation of conjunction ones; the use of the axioms $A, B>A \vee B$ and $\neg A, \neg B>\neg(A \wedge B)$, respectively, makes the rule straightforwardly admissible.

\section{Correia's Logic}

In this section we introduce Correia (2014) logics for the concepts of strict and weak full and immediate grounding. We underline that Correia (2014) never mentions the distinction between immediate and mediate grounding and his results concern mediate grounding. Nevertheless, it is quite trivial to see how to adapt his logic and his results to the only case of immediate grounding.

Definition 5.1. Let $\mathcal{L}^{\prime}$ be the language composed by: propositional atoms: p, q, $r, \ldots$; the classical connectives: $\neg, \wedge, \vee$, and the parentheses: (, ). The formulas of the language $\mathcal{L}$ are defined standardly. $M, N, \ldots$ will denote sets of formulas.

The calculus CG (for Correia on grounding) is composed by the rules in Figure 4, which basically correspond to the axioms of the calculus FG.

Definition 5.2. We say that $M$ strictly, fully and immediately ground $A$, in symbols $M \triangleright A$, if, and only if, $M$ and $A$ can be connected by means of one of the rules of the calculus $\mathbf{C G}$.

Definition 5.3. We say that $M$ weakly, fully and immediately ground $A$, in symbols $M \triangleright A$, if, and only if, $A \in M$ or, for some $N \subseteq M, N \triangleright A$.

As Correia (Correia, 2014, p. 8) himself remarks, his notion of weak ground is significantly different from the one that we saw in Fine: while his notion 
obeys weakening, Fine's does not. It has to be said that doubts may be expressed as to whether a notion of grounding should satisfy weakening: indeed it seems intrinsic to the concept of grounding that all grounds are relevant to the conclusion, while weakening notoriously violates this condition.

Let us list some properties of $\triangleright$ and $\triangleright$.

Proposition 5.4. For any $M$ and any $A$,

- if $M \triangleright A$, then $M \neq \emptyset$,

- if $M \triangleright A$, then $A$ is not an atom, nor the negation of an atom,

- It is not the case that $M, A \triangleright A$,

- if $M \triangleright A$, then the complexity of $A$ is strictly greater than the complexity of any member of $M$, following the standard notion of complexity of a formula, ${ }^{2}$ e.g. see Troelstra and Schwichtenberg (1996).

Proposition 5.5. For any $M$ and any $A$,

- if $M \triangleright A$, then $M \neq \emptyset$,

- if $M \triangleright A$ and $A \notin M$, then $A$ is not an atom, nor the negation of an atom,

- $M, A \triangleright A$,

- if $M \triangleright A$, then $M, N \triangleright A$.

Let us now consider the calculus $\mathbf{G} \mathbf{C}^{\prime} ; \mathbf{G C}^{\prime}$ is obtained from $\mathbf{G C}$ by adding the following two rules:

$$
\frac{A, B}{A \vee B} \vee I \quad \frac{\neg A, \neg B}{\neg(A \wedge B)}(\neg \wedge) I
$$

Definition 5.6. $M \triangleright^{\prime} A$, if, and only if, $M$ and $A$ can be connected by means of one of the rules of the calculus $\mathbf{C G}^{\prime}$.

Definition 5.7. $M \triangleright^{\prime} A$, if, and only if, $A \in M$ or, for some $N \subseteq M, N \triangleright^{\prime} A$.

Correia works with the aforementioned notions $\triangleright$ and $\triangleright$; nevertheless he considers $\triangleright^{\prime}$ and $\triangleright^{\prime}$ as two serious alternative logics. While $\triangleright$ and $\triangleright^{\prime}$ clearly do not have the same extension, $\boldsymbol{\nabla}$ and $\boldsymbol{}^{\prime}$ are equivalent.

In the last section we have underlined the importance of the amalgamation rules in Fine's logic. Such a rule turns out to be admissible in the calculus GC $^{\prime}$ with respect to both notions $\triangleright^{\prime}$ and $\boldsymbol{}^{\prime}$. The proof of the admissibility of the rule is analogous to the one that we have sketched in the case of Fine's logics (see Section 4).

\footnotetext{
${ }^{2}$ Let us underline that Correia uses a notion of complexity which is slightly different from the one that can be standardly found in the literature. Nevertheless Claim 4 of Proposition 5.4 holds for both notions.
} 


\section{$6 \quad$ Negative truths}

Only a few logics have been proposed for the concept of grounding; in the last sections we have introduced and illustrated two of the main ones, namely those of Fine and Correia. In this section and following two, we will analyze these logics in detail. Our analysis will not be technical in nature, i.e. we will not discuss the technical virtues and defects of the calculi FG and CG; rather we will focus on the formal principles governing the concept of full and immediate grounding that these calculi propose and we will evaluate them from a prooftheoretic perspective. We will argue that some of these principles need to be changed, while other principles need to be added. More precisely, while in this section we will discuss principles concerning grounding and negation, in the next section we will discuss principles concerning grounding and disjunction; finally in Section 8 we will discuss principles concerning grounding and truths that are equivalent under applications of associativity and commutativity of conjunction and disjunction.

Let us start by focussing on the axiom $\neg A, \neg B>\neg(A \vee B)$ of $\mathbf{F G}$ and the rule $(\neg \vee) I$ of $\mathbf{C G}$. The axiom and the rule basically say the same thing: the strict, full and immediate grounds of any formula of the form $\neg(A \vee B)$ are $\neg A$

and $\neg B$. Let us confront this statement with some concrete examples. Let us consider the truth "it is not the case that Mary is tall or thin" and let us ask ourselves what the strict, full and immediate grounds of this truth are. The natural answer to such a question is "Mary is not tall" and "Mary is not thin": both these truths seem to be the (full and immediate) reasons of the truth "it is not the case that Mary is tall or thin"; thus the answer agrees with the logics FG and CG.

Let us now take another example. Consider the truth "it is not the case that Mary is not tall or not thin": what are its strict, full and immediate grounds? A little reflection suffices to realize that in this case the natural answer is "Mary is tall" and "Mary is thin": "Mary is tall" and "Mary is thin" seem indeed to be the (full and immediate) reasons why "it is not the case that Mary is not tall or not thin" is true. In this case the answer differs from that given by the logics FG and CG: according to their axioms and rules, the strict, full and immediate grounds of "it is not the case that Mary is not tall or not thin" are "it is not the case that Mary is not tall" and "it is not the case that Mary is not thin". So in this case intuition seems to suggest that the rules of Fine and Correia are not correct. Since intuitions might be revealing but are not always trustworthy, let us develop a more accurate analysis of the situation.

In order to be brief but precise, let us first of all use the following formalization:

- "Mary is tall" and "Mary is thin" are formalized by $p$ and $q$, respectively,

- "it is not the case that Mary is not tall" and "it is not the case that Mary is not thin" are formalized by $\neg \neg p, \neg \neg q$, respectively,

- "it is not the case that Mary is not tall or not thin" is formalized by the 
formula $\neg(\neg p \vee \neg q)$.

The issue here is to establish what the strict, full and immediate grounds of $\neg(\neg p \vee \neg q)$ are: $p$ and $q$ or $\neg \neg p$ and $\neg \neg q$.

One good reason for choosing $p$ and $q$ over $\neg \neg p, \neg \neg q$ appeals to a question of complexity. Indeed, if we consider the standard way of measuring the complexity of a formula ${ }^{3}$, but also, and most importantly, if we take into account the measure of complexity put forward by Correia (2014), $p$ and $q$ are less complex than $\neg \neg p$ and $\neg \neg q$. Grounding is essentially an explanatory relation and, as Ockham teaches us, explanans that are less complex are to be preferred over explanans that are more complex. Thus $p$ and $q$ are the strict, full and immediate grounds of $\neg(\neg p \vee \neg q)$, contrary to what logics FG and CG claim.

This argument for opting for $p$ and $q$ over $\neg \neg p, \neg \neg q$ seems correct; nevertheless, in view of the distinctions that we have introduced in Section 2, one could easily reply to it as follows. $p$ and $q$ certainly represent the grounds for the truth $\neg(\neg p \vee \neg q)$; however they do not substitute $\neg \neg p$ and $\neg \neg q$ in the role of strict, full and immediate grounds of $\neg(\neg p \vee \neg q)$, since they are not its strict, full and immediate grounds, rather they are its strict, full and mediate grounds. To see this, let us consider the calculus CG (we could have equivalently used the calculus FG); in this calculus we can construct the following grounding chain, that we will call $g c$ :

$$
\frac{\frac{p}{\neg \neg p} \quad \frac{q}{\neg \neg q}}{\neg(\neg p \vee \neg q)}
$$

Such a chain evidently shows ${ }^{4}$ that $p$ and $q$ are the (strict, full and) mediate grounds of $\neg(\neg p \vee \neg q)$. Thus $p$ and $q$ are certainly less complex than $\neg \neg p$ and $\neg \neg q$ according to two significant measures of complexity; nevertheless they are not the full and immediate grounds of $\neg(\neg p \vee \neg q)$; they are indeed its full and mediate grounds.

Let us dwell for a moment on the grounding chain $g c$ that we have constructed above. Even at the first glance, this chain has the following striking property: two formulas are introduced, namely $\neg \neg p$ and $\neg \neg q$, which then disappear in the conclusion, i.e. neither one nor the other is a subformula of the formula that appears in the conclusion. In the light of what has been said in Section 3, this fact is alarming. Even if we do not have a rigorous definition of the notion of subformula for the grounding framework and thus we do not have a proper notion of analyticity for grounding chains ${ }^{5}$, a phenomenon of this sort

\footnotetext{
${ }^{3}$ The complexity of a formula $\mathrm{A}, \mathrm{cm}(A)$, is inductively defined in the following way: $\mathrm{cm}(p)$ $=0, c m(\neg A)=c m(A)+1$ and $c m(A \circ B)=c m(A)+c m(B)+1$, where $\circ=\wedge, \vee$. E.g. see Troelstra and Schwichtenberg (1996).

${ }^{4}$ Even if we did not formally introduce the notions of grounding chain and of full and mediate grounding in the calculus CG (for a detailed description, see Correia (2014)), it is quite straightforward to understand them.

${ }^{5}$ This paper is not the place for introducing a rigorous notion of analyticity for the grounding framework since it only concerns a critical analysis of the logics of Fine and Correia. We nevertheless believe that such a task should be seriously taken into account in the studies dedicated to the concept of grounding.
} 
cannot but be suspicious: it is the typical example of loss of analyticity in the standard calculus of natural deduction. In order to get a better grasp of the situation, we can thus proceed in the following way. We try to reconstruct the steps from $p$ and $q$ to $\neg \neg p$ and $\neg \neg q$ to $\neg(\neg p \vee \neg q)$ in the calculus of natural deduction. If we come up with a non-analytic derivation, then this will be a clear sign of the fact that $g c$ is a non-analytic grounding chain. ${ }^{6}$ We have the following derivation:

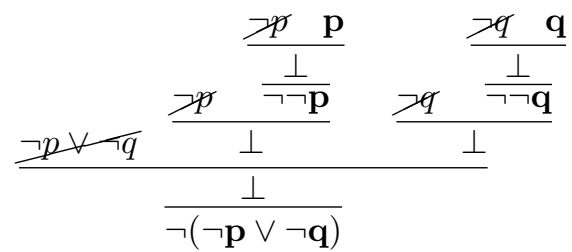

Such a derivation contains a cut, namely the introduction of a negation and its successive elimination; a cut is nothing but the formal counterpart of a nonanalytic inferential step. Thus the derivation that leads from $p$ and $q$ to $\neg \neg p$ and $\neg \neg q$ to $\neg(\neg p \vee \neg q)$ in the calculus of natural deduction is non-analytic; and hence so is the chain $g c$ that we have constructed above. It is very important to note that no derivation containing a cut arises when we reproduce in the calculus of natural deduction the grounding steps from $p$ and $q$ to $\neg(\neg p \vee \neg q)$, and from $\neg p$ and $\neg q$ to $\neg(p \vee q)$ : these grounding steps are those that we previously classified as valid.

We can take this situation to be the formal counterpart of the intuitions that we have exposed at the beginning of the section. Given the truth "it is not the case that Mary is not tall or not thin", it seems natural to claim that its full and immediate grounds are the truths "Mary is tall" and "Mary is thin". As a matter of fact, even formally the step from "Mary is tall" and "Mary is thin" to "it is not the case that Mary is not tall or not thin" corresponds to a normal derivation in the calculus of natural deduction (and thus, most likely, to an analytic grounding step). By contrast, if we try to claim that the truths "Mary is tall" and "Mary is thin" are instead the full and mediate grounds of the truth "it is not the case that Mary is not tall or not thin", since the full and immediate grounds of this latter truth are the truths "it is not the case that Mary is not tall" and "it is not the case that Mary is not thin", not only do we seem to be making an artificial construction, but also, at the formal level, we are compelled to accept a non-analytic grounding chain (because we have a non-normal derivation in the calculus of natural deduction).

Therefore it seems reasonable to conclude that not all instances of the axiom $\neg A, \neg B>\neg(A \vee B)$ of $\mathbf{F G}$ and the rule $(\neg \vee) I$ of $\mathbf{C G}$ are correct; in particular the only case where they work adequately is when neither in $A$ nor in $B$ the principal connective is a negation; the other cases need a more careful treatment.

\footnotetext{
${ }^{6}$ Under the assumption that grounding proofs are nothing but particular type of derivations, if the derivation that is behind a specific grounding chain is not analytic, then the grounding chain is not analytic too.
} 
Similar conclusions hold for the axioms $\neg A, \neg B>\neg(A \wedge B), \neg A>\neg(A \vee B)$, $\neg B>\neg(A \vee B)$ and all the rules $(\neg \wedge) I$.

\section{Disjunctive truths (and the full-partial dis- tinction)}

We will use this section to point out some flaws of both the distinction between full and partial grounding, as it has been introduced in Section 2, and the axioms of FG concerning disjunction and grounding. As one shall argue, these criticisms are interrelated.

Before starting, let us emphasize two things. First of all, we point out that anything that will be said of the axioms of $\mathbf{F G}$ also holds for the corresponding rules of the system $\mathbf{C G}^{\prime}$. Secondly, we stress that the criticisms directed to the axioms of FG concerning disjunction and grounding also hold for those concerning negation of conjunction. For the sake of brevity, we will only consider the former and not the latter.

Let us begin by noting a significant mismatch between the full-partial distinction as introduced in Section 2 and certain axioms of FG that are supposed to reflect this distinction. For this, let us consider the three axioms of FG describing disjunction and grounding, namely $A>A \vee B, B>A \vee B, A, B$ $>A \vee B$ and let us distinguish two ways of reading the full-partial distinction as it has been introduced in Section 2: a way that might be called minimal and a way that might be called non-minimal. According to the minimal way of reading the full-partial distinction, to give the full grounds of $A \vee B$ amounts to give exactly sufficient conditions for the truth $A \vee B$ and nothing more. In this context, while the axioms $A>A \vee B, B>A \vee B$ properly reflect the relation of full grounding, the third axiom does not reflect this distinction at all: $A$ and $B$ together are indeed more than sufficient for explaining the truth $A \vee B$. Let us then turn to the non-minimal way of reading the full-partial distinction. According to the non-minimal way of reading the full-partial distinction, to give the full grounds of $A \vee B$ may include giving even more than what is strictly sufficient for the truth $A \vee B$. In this context the three axioms $A>A \vee B, B$ $>A \vee B, A, B>A \vee B$ all reflect the relation of full grounding. The problem in this case is rather the following: if we accept this non-minimal reading of the distinction full-partial, then we should also be willing to accept axioms of the form $A, C>A \vee B, B, C>A \vee B, A, B, C>A \vee B \ldots$ : each of these axioms indeed provides more than sufficient conditions for the truth $A \vee B$ and has thus the same status as the three axioms $A>A \vee B, B>A \vee B, A, B>A \vee B$. Under the non-minimal reading of the full-partial distinction, the logic for the notion of full and immediate ground should thus include not only the axioms $A$ $>A \vee B, B>A \vee B, A, B>A \vee B$, but also axioms of the form $A, C>A \vee B$, $B, C>A \vee B, A, B, C>A \vee B$... Such a conclusion is of course unacceptable and thus the non-minimal reading of the full-partial distinction is to be rejected. We should thus stick to the minimal reading of the full-partial distinction and 
find a solution for the tension between such a reading and the axiom $A, B>$ $A \vee B$. More precisely, the issue is that of understanding whether we should keep the full-partial definition (under a minimal reading) and reject the axiom, or whether, on the contrary, it is preferable to keep the axiom and reject the definition (under a minimal reading); in what follows we give an argument in favor of the second option.

For this let us consider a world where both $A$ and $B$ are true. If we follow the distinction full-partial, as it has been defined in Section 2, and the axioms that properly reflect it, $A$ (or similarly $B$ ) is, by itself, the strict full and immediate ground of $A \vee B$. Is this really the case? Generally speaking, the grounding relation is indeed a relation of strong connection and authentic dependence between premisses and conclusion. This is even more true of the relation of full and immediate grounding that is, among the four relations of grounding, the most stringent and fundamental (e.g. see Tatzel (2002)). Thus, we would like to claim that, in a full and immediate grounding relation, if the antecedent were not true, the consequent would not be true either. Unfortunately, this is not what happens with the full and immediate grounding relation that we have just stated: in a world where both $A$ and $B$ are true, the fact that $A$ is the full and immediate ground of $A \vee B$ does not entail that, if $A$ were not true, $A \vee B$ would not be true either, since $A \vee B$ would still be true because of $B$. Thus, in a world where both $A$ and $B$ are true, $A$ does not seem to share such a strict connection with the truth $A \vee B$; in other words, $A$ does not seem to be the strict, full and immediate ground of $A \vee B$. In such a situation it seems rather that both $A$ and $B$ are the full and immediate grounds of $A \vee B$ : they both contribute, and hence explain, the truth $A \vee B$. Moreover, they stand in authentic dependency with $A \vee B$ : if both $A$ and $B$ were not true, $A \vee B$ would not be true either. Therefore, in a world where both $A$ and $B$ are true, the strict, full and immediate grounding relation for the truth $A \vee B$ is properly described by the axiom of the form $A, B>A \vee B$. This seems to us as a very good reason for keeping such an axiom and, in view of its incompatibility with the full-partial distinction, for rethinking this distinction.

The second criticism that we propose in this section has to do with something that has already been mentioned in Section 2. In that section we introduced two distinctions, full-partial and immediate-mediate, that combined together identify four different types of grounding: full and immediate, full and mediate, partial and immediate and partial and mediate. The reason for introducing these four types of grounding cannot but be classificatory; in the same way as, once one has introduced the concept of natural number, then each number is either even or odd, thus, once one has introduced the concept of grounding, any grounding relation would better be of one, and only one, of the four types of grounding relation mentioned above. This is not what happens with our classification: given a set of truths $M$ and a truth $A$ that stand in a grounding relation, this grounding relation can be of more than one type; actually, it can be of all four types at the same time, as is the case for $A$ and $A \vee(A \vee A)$ (see Section 2 ). Such a fact should invite a serious reconsideration of our distinctions: if the limits that they describe are so wide, does this not weaken their power? Clearly, 
we would conceptually gain much if we could draw the distinctions between full and partial and immediate and mediate in such a way that the four types of grounding never overlap (or at least as little as possible).

Note that such a criticism of the full-partial and immediate-mediate distinctions also holds for certain axioms and rules of the logic of Fine (2012a). ${ }^{7}$ Consider for example the following two axioms that describe the full and immediate grounds of a disjunctive truth, $A>A \vee B$ and $B>A \vee B$, and suppose that one adds the symbol $\succ$ for partial and immediate ground. The complete version of the logic of Fine (2012a) contains this rule:

$$
\frac{M, A>C}{A \succ C}
$$

which allows us to infer the following theorems: $A \succ A \vee B$ and $B \succ A \vee$ $B$. Thus, in the logic of Fine it holds that $A$, or equivalently $B$, is at the same time the full and the partial, immediate grounding of the truth $A \vee B$. The same ambiguity, which has been previously pointed out for the informal full-partial and immediate-mediate distinctions, can thus now be noted for the formal principles that govern the logic introduced in Fine (2012a). This suggests that certain axioms and rules of this logic should be also carefully reconsidered.

The full-partial and immediate-mediate distinctions have been related to the notion of grounding since (at least) the XIX century. Bolzano (1996) introduced them for the first time and defined them in a way that overcomes the problems outlined in this section. Despite the fact that nowadays most philosophers have emphatically neglected Bolzano's work and embraced Fine's demarcations, the criticisms that have been put forward in this section suggest that such a choice has not necessarily been a good one. We think that a renewed attention should be dedicated to the reflexions of the great Bohemian philosopher and clearer and more precise definitions of the full-partial (and immediate-mediate) distinction should be investigated.

\section{Associatively and commutatively equivalent truths}

We use this section to discuss some formal principles concerning grounding that are neglected by the logics of Fine and Correia, but that, we will argue, represent important features of the concept of grounding.

Let us start considering the truth "Mary is tall and thin, and blond" and let us recall the strict, full and immediate grounds of this truth according to the logics FG and CG: "Mary is tall and thin" and "Mary is blond" very naturally fill this role. Let us now consider the truths "Mary is thin and tall" and "Mary is blond" and let us consider the relation between these truths and the truth

\footnotetext{
${ }^{7}$ This is not immediately clear from what we have presented in Section 4 , since in that section we have restricted ourselves to the only case of full and immediate grounding. A quick look to Fine (2012a) will be enough to verify what we are saying.
} 
"Mary is tall and thin, and blond". Without any doubt one would like to say that "Mary is thin and tall" and "Mary is blond" are the grounds of "Mary is tall and thin, and blond": whatever conception of ground one might have, such a conception must contain "Mary is thin and tall", "Mary is blond" and "Mary is tall and thin, and blond" amongst the truths connected by the grounding concept; otherwise, it can be seriously doubted that we understand each other when talking about grounding.

Let us now try to understand what kind of grounding relation the truths "Mary is thin and tall", "Mary is blond" and "Mary is tall and thin, and blond" enjoy. As long as we think that "Mary is tall and thin" and "Mary is blond" are the strict, full and immediate grounds of "Mary is tall and thin, and blond", we should think the same of "Mary is thin and tall" and "Mary is blond": there is indeed no difference between these two pairs of truths that could possibly lead us to classify the former according to one type of grounding, and the latter according to another type.

Let us formalize what we have said in the following way:

- "Mary is tall and thin" and "Mary is blond" are formalized by $p \wedge q$ and $r$,

- "Mary is thin and tall" and "Mary is blond" are formalized by $q \wedge p$ and $r$,

- "Mary is tall and thin, and blond" is formalized by $(p \wedge q) \wedge r$.

According to the logics FG and $\mathbf{C G}, p \wedge q$ and $r$ are the full and immediate grounds of $(p \wedge q) \wedge r$ : in the former case this is an instance of one of the axioms of Figure 1, in the latter case this is due to the rule $\wedge I$. On the other hand, in neither of the logics FG and CG, are $q \wedge p$ and $r$ the full and immediate grounds of $(p \wedge q) \wedge r$ : indeed in the former case, there is no axiom the describes such a relation, in the latter case there is no rule that allows us to infer such a link. ${ }^{8}$ Given what we have said in the previous paragraph, this is an important defect of the logics FG and CG: by means of these logics we should indeed be able to prove all correct grounding relations, so the lack of the one holding amongst $q \wedge p$ and $r$ and $(p \wedge q) \wedge r$ counts as a serious flaw of these theories.

Let us note that the problem raised by the relation among the truths $q \wedge p$, $r$ and $(p \wedge q) \wedge r$ for the logics FG and CG is just a small example of a more general situation. In the rest of the section we describe in detail this situation. Consider a truth $A$; $A$ is said to be a-c equiv to another truth $B$ if, and only if, $B$ can be obtained from $A$ by applications of associativity and commutativity of conjunction and disjunction. ${ }^{9}$ For example, if $A$ is of the form $E \wedge F$, then the formula $F \wedge E$ is a-c equiv to it. If $A$ is of the form $\neg((B \vee C) \wedge(D \vee F))$ the formulas $\neg((C \vee B) \wedge(D \vee F)), \neg((B \vee C) \wedge(F \vee D)), \neg((C \vee B) \wedge(F \vee D))$ are $a-c$ equiv to it. If $A$ is of the form $((B \vee C) \vee(D \vee F))$, then the formulas

\footnotetext{
${ }^{8}$ Worse, if we are not mistaken, in the logics FG and CG $q \wedge p$ and $r$ cannot be shown to be the grounds of $(p \wedge q) \wedge r$ tout court.

${ }^{9}$ We omit the formal definition of this notion for the sake of brevity.
} 
$((B \vee D) \vee(C \vee F)),((D \vee B) \vee(F \vee C)),((B \vee F) \vee(D \vee C))$ are all a-c equiv to it. The central remark here is the following. If grounding is defined as an explanatory objective relation amongst truths, then the order and the way in which these truths are arranged should not condition the grounding relation in any way: neither in the very existence of the grounding relation nor in the type this grounding relation is. Therefore, not only the full and immediate grounds of $A$ are the truths $M$ indicated by the axioms of Figure 1. of FG (or, equivalently, by the rules of Figure 4. of the logic $\mathbf{C G}$ ), but also, for any set of truths $N$ and any truth $C$ such that $C$ is $a-c$ equiv to $A$, if $\mathrm{N}$ is the full and immediate ground of $C$ according to the axioms of Figure 1. of FG (or, equivalently, according to the rules of Figure 4 . of the logic $\mathbf{C G}$ ), then $N$ is also the full and immediate ground of $A$.

Let us consider a few examples that support what we have just said. Consider the truths $\neg \neg((p \wedge q) \wedge t)$ and $(p \wedge q) \wedge t$ : according to the logics FG and $\mathbf{C G}$ these truths stand in a full and immediate grounding relation. Consider now the following truths: $(q \wedge p) \wedge t,(p \wedge t) \wedge q,(t \wedge p) \wedge q,(q \wedge t) \wedge p, \ldots$ It seems difficult to support the claim that in a grounding hierarchy $(p \wedge q) \wedge t$ and each of these latter truths occupy different positions. On the contrary, as long as $(p \wedge q) \wedge t$ is a (full and immediate) ground of the truth $\neg \neg((p \wedge q) \wedge t)$, each of these truths $(q \wedge p) \wedge t,(p \wedge t) \wedge q,(t \wedge p) \wedge q,(q \wedge t) \wedge p, \ldots$ should be a full and immediate ground too.

Consider now the truth $((p \vee q) \vee t) \wedge r$; everybody agrees that $((p \vee q) \vee t)$ and $r$ are the full and immediate grounds of this truth. But then everybody should also agree on the fact that $((q \vee p) \vee t)$ and $r$ are the full and immediate grounds of $((p \vee q) \vee t) \wedge r$ too: between $((p \vee q) \vee t)$ and $r$ on the one hand, and $((q \vee p) \vee t)$ and $r$ on the other hand, there is no difference that would justify the claim that the former are (full and immediate) reasons of the truth $((p \vee q) \vee t) \wedge r$, and the latter are not. The same holds for other pairs of truths such as: $((q \vee t) \vee p)$ and $r,((t \vee q) \vee p)$ and $r,((t \vee p) \vee q)$ and $r \ldots$ : we cannot at the same time claim that $((p \vee q) \vee t)$ and $r$ are the full and immediate grounds of $((p \vee q) \vee t) \wedge r$, and each of these pairs is not.

Note that at a first glance one might be lead to think that the associativity of conjunction and disjunction is less natural than the commutativity of conjunction and disjunction in the classification of truths linked by the full and immediate grounding relation. Under such a perspective, some doubts might arise about what has been argued for in this section. In order to remove such doubts, let us add the following observations. Instead of considering the relation of grounding from the grounds perspective, let us consider it from the perspective of the conclusions. Consider indeed two truths like $(A \vee B) \vee C$ and $A \vee(B \vee C)$. These two truths are logically equivalent; plus they are made up by the same connectives used a same number of times. Finally, they are composed by the same propositional atoms. In a grounding hierarchy, which has a pure ontological nature, these two truths should be considered as identical. Indeed the only difference between them is the order in which the propositional atoms are grouped; such a difference is certainly significant from an epistemic point of view but basically irrelevant from a metaphysical one. From this latter point of 
view, there is no important difference between the two truths and therefore it seems hard to deny that they do not have the same full and immediate grounds.

We can thus conclude that the relation of full and immediate grounding is in a certain sense closed under associativity and commutativity of conjunction and disjunction and this characteristic is neglected by the logics FG and CG. Axioms and rules should be added to take into account these important grounding relations.

\section{Conclusions}

In this paper we have focussed on the two most recent logics of grounding: the one introduced by Fine in 2012, and the one introduced by Correia in 2014. Both these logics have the great merit and the undoubted virtue of contributing to the formal study of the principles concerning the grounding relation: our intuitions on grounding are systematized in a rigorous and precise treatment. In this paper we have tried to show in what way the formal principles put forward by Fine and Correia should be in some cases changed, in other cases enhanced. The intention has been to lay the basis for future debates and developments.

\section{References}

Avron, A. (1991). Simple consequence relation. Information and Comutation, 92:105-139.

Batchelor, R. (2010). Grounds and consequences. Grazer Philosophische Studien, 80:65-77.

Betti, A. (2010). Explanation in metaphysics and Bolzano's theory of ground and consequence. Logique et analyse, 211:281-316.

Bolzano, B. (1973). Theory of Science: A Selection, with an Introduction. D. Riedel, Dordrecht.

Bolzano, B. (1996). Contributions to a more well founded presentation of mathematics. In Ewald, W. B., editor, From Kant to Hilbert : A source book in the foundations of mathematics, pages 176-224. Oxford University Press, Oxford.

Casari, E. (1987). Matematica e verità. Rivista di Filosofia, 78(3):329-350.

Correia, F. (2010). Grounding and truth-functions. Logique et Analyse, 53(211):251-79.

Correia, F. (2014). Logical grounds. Review of Symbolic Logic, 7(1):31-59.

Correia, F. and Schnieder, B. (2012). Grounding: an opinionated introduction. In Correia, F. and Schnieder, B., editors, Metaphysical grounding, pages 1-36. Cambridge University Press, Cambridge. 
Daily, C. (2012). Scepticism about grounding. In Correia, F. and Schnieder, B., editors, Metaphysical grounding, pages 81-100. Cambridge University Press, Cambridge.

de Rosset, L. (2013). What is weak ground? Essays in Philosophy, 14(1):7-18.

Fine, K. (2012a). Guide to ground. In Correia, F. and Schnieder, B., editors, Metaphysical grounding, pages 37-80. Cambridge University Press, Cambridge.

Fine, K. (2012b). The pure logic of ground. Review of Symbolic Logic, 25(1):125.

Gentzen, G. (1935). Untersuchungen über das logische schließen. Mathematische Zeitschrift, 39:176-210.

Indrezejczak, A. (1997). Generalised sequent calculus for propositional modal logics. Logica Trianguli, 1:15-31.

Paoli, F. (1991). Bolzano e le dimostrazioni matematiche. Rivista di Filosofia, LXXXIII:221-242.

Poggiolesi, F. (2008). A cut-free simple sequent calculus for modal logic S5. Review of Symbolic Logic, 1:3-15.

Poggiolesi, F. (2010). Gentzen Calculi for Modal Propositional Logic. Springer.

Poggiolesi, F. (2012). On the importance of being analytic. The paradigmatic case of the logic of proofs. Logique et Analyse, 219:443-461.

Poggiolesi, F. (2013). From a single agent to multi-agent via hypersequents. Logica Universalis, 3:443-461.

Prawitz, D. (1965). Natural deduction. A proof theoretic study. Almquist and Wiskell.

Rumberg, A. (2013). Bolzano's theory of grounding against the background of normal proofs. Review of Symbolic Logic, 6(3):424-459.

Schaffer, J. (2009). On what grounds what. Metametaphysics, pages 347-383.

Schnieder, B. (2011). A logic for 'Because'. The Review of Symbolic Logic, 4(03):445-465.

Sebestik, J. (1992). Logique et mathematique chez Bernard Bolzano. J. Vrin.

Tatzel, A. (2001). Proving and grounding: BolzanoÔs theory of grounding and gentzenÔs normal proofs. History and Philosophy of Logic, to appear, 1:1-28.

Tatzel, A. (2002). Bolzano's theory of ground and consequence. Notre Dame Journal of Formal Logic, 43(1):1-25.

Troelstra, A. S. and Schwichtenberg, H. (1996). Basic Proof Theory. Cambridge University Press. 